Dr. McCrae's induction will remind physicians of their obligation to serve their country and the global community.

Vivian McAlister, MD

Coeditor, Canadian Fournal of Surgery

Competing interests: None declared.

DOI: $10.1503 /$ cjs. 003215

\section{References}

1. Rotstein O, Greig P. Dr Bernard Langer - inductee into the Canadian Medical Hall of Fame. Can 7 Surg 2015;58:80-2.

2. Prescott JF. The extensive medical writings of soldier-poet John McCrae. CMA7 1980;122:113-4.

3. Ullah SM, Grant RC, Johnson M, et al. Scarpa's fascia and clinical signs: the role of the membranous superficial fascia in the eponymous clinical signs of retroperitoneal catastrophe. Ann R Coll Surg Engl 2013;95:519-22.

\title{
La commémoration médicale
}

e besoin de commémoration est fort en médecine, particulièrement chez nos collègues chirurgiens. Il persiste malgré les modes, quelle que soit l'époque. C'est peut-être le désir de passer à l'histoire qui pousse les chirurgiens à travailler plus fort et plus longtemps que leurs pairs dans la société. La commémoration médicale, soit la volonté qu'a une société de perpétuer le souvenir d'un médecin, est, quant à elle, un mystère. Elle semble être aléatoire et se révèle souvent inexacte. Elle est discrètement vénérée, mais ne doit pas être recherchée.

Le 23 avril 2015, 100 ans exactement après la seconde bataille d'Ypres, John McCrae sera intronisé au Temple de la renommée médicale canadienne. Sa candidature avait été soumise 2 fois auparavant pour occuper la seule place réservée chaque année à une intronisation posthume. Le $\mathrm{D}^{\mathrm{r}}$ Bernard Langer compte parmi les autres lauréats de cette année, qui sont heureusement toujours en vie. Le $\mathrm{D}^{\mathrm{r}}$ Langer, ancien chef du Département de chirurgie de l'Université de Toronto, est reconnu pour ses contributions à l'éducation médicale, à la recherche et à la sécurité des patients ${ }^{1}$. John McCrae, révéré pour son poème intitulé «Au champ d'honneur », a probablement été sélectionné cette fois-ci parce que son service en temps de guerre a été placé dans le contexte du niveau avancé de sa pratique médicale au moment de son enrôlement. Les publications médicales du $\mathrm{D}^{\mathrm{r}} \mathrm{McCrae}$ démontrent son rôle de pionnier dans les domaines des maladies infectieuses et de la pathologie anatomique ${ }^{2}$. Il aurait été intronisé en qualité de bâtisseur s'il n'était pas mort pendant la guerre. Cette distinction ajoute peu à la renommée du $\mathrm{D}^{\mathrm{r}}$ John McCrae : des écoles et des prix ont déjà été nommés en son honneur et le gouvernement du Canada l'a désigné « personne d'importance historique nationale » il y a 70 ans. L'intronisation du $\mathrm{D}^{\mathrm{r}} \mathrm{John}$ McCrae au Temple de la renommée médicale canadienne est notre façon de rapatrier au sein de la médecine le soldat-poète. L'objectif de cet article est d'honorer ces médecins et d'examiner le rôle de la commémoration en médecine.

La commémoration, qui remonte aux sagas préhistoriques transmises oralement, s'est manifestée plus tard dans les statues de l'époque classique et dans les monuments de l'époque victorienne. La commémoration était réservée à l'élite dirigeante et son expression ultime était l'élection au panthéon. Le Temple de la renommée est un panthéon moderne auquel l'accès a été démocratisé.

Au $19 \mathrm{e}$ siècle, avec les progrès en bactériologie, de nombreux organismes jusqu'alors inconnus ont été désignés du nom de leur découvreur. Cette pratique, semblable à celle des explorateurs qui baptisaient de nouveaux lieux, laisse entendre que les découvreurs conservent des droits d'appellation. Ce n'est pas le cas en médecine. Contrairement au domaine de la géographie, revendiquer un nom pour une découverte médicale ou une invention ou donner un nom en l'honneur d'un tiers, tel que celui d'un commanditaire ou d'un membre de la famille royale, n'est pas bien vu. Les universités peuvent offrir des droits d'appellation en échange de dons, mais cette pratique relève beaucoup plus de la commandite de complexes sportifs que de la médecine. Aucune de ces 2 formes d'appellation ne constitue une commémoration médicale.

Les désignations éponymes, souvent considérées comme une forme prisée de commémoration, peuvent 
faciliter la mémorisation et la communication. Cela est particulièrement vrai en ce qui concerne les signes cliniques - un ensemble d'observations qui conduit le médecin à un diagnostic instantané et résulte en une confiance éternelle du patient. De nombreuses désignations éponymes sont toutefois inexactes. Par exemple, même si Thomas Cullen a attribué la description des taches périombilicales à Joseph Ransohoff, nous nous en souvenons comme du signe de Cullen ${ }^{3}$. Les appellations éponymes données aux procédures chirurgicales sont encore plus inexactes. Les interventions novatrices sont fondées sur des pratiques antérieures. Allen Whipple n'a ni décrit, ni été le premier à effectuer l'intervention connue sous le nom d'« opération de Whipple ». Malgré ces inexactitudes, il n'y a aucune contestation ni aucune campagne pour corriger ces erreurs. Cela est attribuable au fait que les désignations éponymes sont inspirées du nom de la personne qui a popularisé quelque chose plutôt que sur le nom de la personne qui a découvert ou inventé la chose en question. Elles sont utilisées seulement si elles facilitent la communication, ce qui explique pourquoi la pancréaticoduodénectomie se nomme «opération de Whipple » alors que la greffe de foie ne se nomme pas « opération de Starzl». L'attribution d'une désignation éponyme n'est donc pas un acte de commémoration.

La commémoration est inhérente à la formation en chirurgie, dans laquelle non seulement nous enseignons une procédure ou une facette du savoir, mais aussi la façon dont elle a été développée. En recherche, cela se traduit par une référence ou par un renvoi aux connais- sances antérieures. Ce sont aujourd'hui les formes privilégiées de commémoration parce qu'elles reconnaissent l'acte plutôt que la personne. Les statues, bustes et portraits des pionniers de la médecine ont été retirés de nos hôpitaux. La reconnaissance de l'individu a toutefois un rôle à jouer. Certains signes indiquent que l'art du portrait revient à la mode, et l'impression tridimensionnelle pourrait rendre la sculpture plus populaire.

Le public cible du Temple de la renommée médicale canadienne est l'ensemble de la société, et particulièrement les jeunes parce que le véritable objectif de la commémoration est d'inspirer. L'intronisation du $\mathrm{D}^{\mathrm{r}}$ Langer inspirera les élèves à choisir une carrière en chirurgie et à imiter ses réalisations et celle du $\mathrm{D}^{\mathrm{r}} \mathrm{McCrae}$ rappellera aux médecins leur devoir de servir leur pays et la communauté mondiale.

Vivian McAlister, MD

Co-rédacteur, fournal canadien de chirurgie

Intérêts concurrents : Aucuns déclaré.

DOI: $10.1503 /$ cjs. 003515

\section{Références}

1. Rotstein O, Greig P. Dr Bernard Langer - inductee into the Canadian Medical Hall of Fame. Can 7 Surg 2015;58:80-2.

2. Prescott JF. The extensive medical writings of soldier-poet John McCrae. CMA7 1980;122:113-4.

3. Ullah SM, Grant RC, Johnson M, et al. Scarpa's fascia and clinical signs: the role of the membranous superficial fascia in the eponymous clinical signs of retroperitoneal catastrophe. Ann R Coll Surg Engl 2013;95:519-22. 